\title{
Fuzzy Similarity in Multicriteria Decision-Making Problem Applied to Supplier Evaluation and Selection in Supply Chain Management
}

\author{
Pasi Luukka \\ Laboratory of Applied Mathematics, Lappeenranta University of Technology, P.O. Box 20, 53851 Lappeenranta, Finland \\ Correspondence should be addressed to Pasi Luukka, pasi.luukka@lut.fi \\ Received 5 August 2011; Accepted 27 November 2011 \\ Academic Editor: Cyrille Bertelle \\ Copyright (C) 2011 Pasi Luukka. This is an open access article distributed under the Creative Commons Attribution License, which \\ permits unrestricted use, distribution, and reproduction in any medium, provided the original work is properly cited.

\begin{abstract}
It is proposed to use fuzzy similarity in fuzzy decision-making approach to deal with the supplier selection problem in supply chain system. According to the concept of fuzzy TOPSIS earlier methods use closeness coefficient which is defined to determine the ranking order of all suppliers by calculating the distances to both fuzzy positive-ideal solution (FPIS) and fuzzy negative-ideal solution (FNIS) simultaneously. In this paper we propose a new method by doing the ranking using similarity. New proposed method can do ranking with less computations than original fuzzy TOPSIS. We also propose three different cases for selection of FPIS and FNIS and compare closeness coefficient criteria and fuzzy similarity criteria. Numerical example is used to demonstrate the process. Results show that the proposed model is well suited for multiple criteria decision-making for supplier selection. In this paper we also show that the evaluation of the supplier using traditional fuzzy TOPSIS depends highly on FPIS and FNIS, and one needs to select suitable fuzzy ideal solution to get reasonable evaluation.
\end{abstract}

\section{Introduction}

The overall objective of supplier selection process is to reduce purchase risk, maximize overall value to the purchaser, and build the closeness and long-term relationships between buyers and suppliers [1]. With the globalization of the economic markets and the development of information technology, many companies consider that a well-designed and implemented supply chain management (SCM) system is an important tool for increasing competitive advantage [2].

Previously, many methods have been proposed to solve the supplier selection problem, some of the popular ones being the linear weighting methods (LW) $[3,4]$, the analytic hierarchy process (AHP) $[5,6]$, the analytic network process [7], total cost approaches $[8,9]$, and mathematical programming (MP) techniques $[10,11]$. However, several influence factors are often not taken into account in the decision-making process, such as incomplete information $[12,13]$, additional qualitative criteria, and imprecision preferences. A strategic approach towards supplier selection often involves multiple criteria [14] and several decisionmakers $[15,16]$, and decision-making is often influenced by uncertainty in practice. Supplier selection may involve several and different types of criteria, combination of different decision models, group decision-making, and various forms of uncertainty. Technique for order performance by similarity to ideal solution (TOPSIS) [17], which is one of the known classical MCDM methods, may provide the basis for developing supplier selection models that can effectively deal with these properties. It bases upon the concept that the chosen alternative should have the shortest distance from the positive ideal solution (PIS) and the farthest from the negative ideal solution (NIS). TOPSIS was further extended to fuzzy environment where linguistic assessments could be used instead of numerical values by Chen [18]. This method allows to consider the fuzziness in the decision data and group decision-making process, where linguistic variables are used to assess the weights of all criteria and the ratings of each alternative with respect to each criterion. In a paper by Chen et al. [19] this approach was further applied to supplier evaluation and selection in supply chain management. In this 
paper, this fuzzy TOPSIS described in [19] is extended to use fuzzy similarity [20]. In fuzzy TOPSIS a vertex method is applied to calculate the distance between two fuzzy ratings. Using this vertex method, they calculate the distance of each alternative from fuzzy positive ideal solution (FPIS) and fuzzy negative ideal solution (NPIS), respectively. After this a closeness coefficient of each alternative is defined to determine the ranking order of all alternatives. The higher value of closeness coefficient indicates that an alternative is closer to FPIS and farther from FNIS. By using fuzzy similarity we are replacing this vertex method for calculating distance by using fuzzy similarity to calculate similarity between two fuzzy ratings. By doing this we can use similarity value gained using proposed method and we do not need to calculate closeness coefficient to get to the result. Second we also study three different alternatives for selecting FPIS and also FNIS in fuzzy TOPSIS and compare how assessments of suppliers are changing depending on the chosen alternatives.

Rest of the paper is organized as follows. Section 2 introduces the basic notations of fuzzy numbers and linguistic variables as well as used fuzzy similarity measure. Section 3 presents fuzzy TOPSIS and how it can be used with fuzzy similarity. There we also introduce three possible FPIS and FNIS criteria. In Section 4 numerical example is used to demonstrate how proposed method can be used and also three criteria for FPIS and FNIS are used to compare the results together with the previously presented fuzzy TOPSIS. Finally some discussion is given at the end of the paper.

\section{Basic Concepts for Generalized Fuzzy Numbers, Linguistic Variables, and Fuzzy Similarity}

Fuzzy logic is a logic that allows vagueness, imprecision, and uncertainty. Zadeh (1965) [21] introduced the concept of fuzzy sets and the respective theory that can be regarded as the extension of the classical set theory. One of the fundamental mathematical constructs is the similarity measure. In the same way as the notion of the fuzzy subset generalizes that of the classical subset, the concept of similarity can be considered as being a multivalued generalization of the classical notion of equivalence [22].

Wei and Chen introduced a fuzzy similarity measure [20] for generalized fuzzy numbers. They tested several similarity methods for generalized fuzzy numbers [23-26] and demonstrated successfully benefits of this measure. First we next briefly review basic concepts of generalized fuzzy numbers [27] and then introduce the similarity measure used later in fuzzy decision-making.

Chen [27] represented a generalized trapezoidal fuzzy number $\tilde{A}$ as $\widetilde{A}=(a, b, c, d ; w)$, where $a, b, c$, and $d$ are real values and $0<w \leq 1$ as shown in Figure 1 .

The membership function $\mu_{\tilde{A}}$ satisfies the following conditions [27]:

(1) $\mu_{\tilde{A}}$ is a continuous mapping from the universe of discourse $X$ to the closed interval in $[0,1]$;

(2) $\mu_{\tilde{A}}=0$, where $-\infty<x \leq a$;

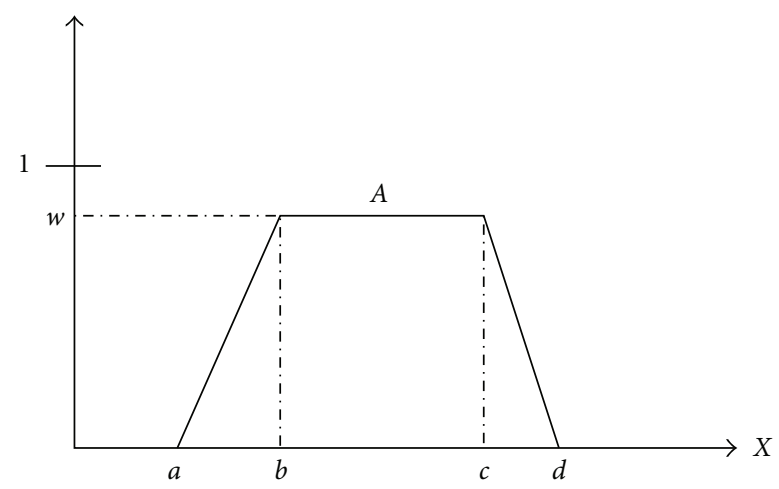

FIgURE 1: A generalized trapezoidal fuzzy number $\tilde{A}=$ $(a, b, c, d ; w)$.

(3) $\mu_{\widetilde{A}}$ is monotonically increasing in $[a, b]$;

(4) $\mu_{\tilde{A}}=w$, where $b \leq x \leq c$;

(5) $\mu_{\tilde{A}}$ is monotonically decreasing in $[c, d]$;

(6) $\mu_{\tilde{A}}=0$, where $d \leq x<\infty$.

Assume that we have two generalized trapezoidal fuzzy numbers $\widetilde{A}$ and $\widetilde{B}$, where $\widetilde{A}=\left(a_{1}, a_{2}, a_{3}, a_{4}, w_{a}\right)$ and $\widetilde{B}=\left(b_{1}, b_{2}\right.$, $\left.b_{3}, b_{4}, w_{b}\right)$.

Now, for example, addition $\oplus$ for the generalized trapezoidal fuzzy numbers is

$$
\begin{aligned}
\widetilde{A} \oplus \widetilde{B} & =\left(a_{1}, a_{2}, a_{3}, a_{4}, w_{A}\right) \oplus\left(b_{1}, b_{2}, b_{3}, b_{4}, w_{b}\right) \\
& =\left(a_{1}+b_{1}, a_{2}+b_{2}, a_{3}+b_{3}, a_{4}+b_{4}, \min \left(w_{a}, w_{b}\right)\right)
\end{aligned}
$$

and subtraction $\ominus$

$$
\begin{aligned}
\tilde{A} \ominus \widetilde{B} & =\left(a_{1}, a_{2}, a_{3}, a_{4}, w_{A}\right) \ominus\left(b_{1}, b_{2}, b_{3}, b_{4}, w_{b}\right) \\
& =\left(a_{1}-b_{4}, a_{2}-b_{3}, a_{3}-c_{2}, a_{4}-b_{1} ; \min \left(w_{a}, w_{b}\right)\right),
\end{aligned}
$$

for example, multiplication $\otimes$ and division $\ominus$ of fuzzy numbers see, for example, [27] or [20].

Notice that these operations for positive trapezoidal fuzzy numbers follows the same arithmetic rules as also earlier used in concept of using fuzzy TOPSIS, for example, in $[18,19]$ with the exception that height of the fuzzy number can be now between $0<w \leq 1$. In this paper we are using normalized fuzzy numbers, where $w=1$, so basically arithmetic rules are the same as in [28]. Basically $w$ can be omitted so that normalized fuzzy number with height equaling one can be represented as $\tilde{A}=(a, b, c, d)$. Here this generalized form for fuzzy numbers is used to show generalized form of fuzzy similarity given by Wei and Chen [20].

Degree of similarity between two generalized fuzzy numbers can be computed as

$$
\begin{aligned}
S(\tilde{A}, \widetilde{B})= & \left(1-\frac{\sum_{i=1}^{4}\left|a_{i}-b_{i}\right|}{4}\right) \\
& \times \frac{\min (P(\widetilde{A}), P(\widetilde{B}))+\min \left(w_{a}, w_{b}\right)}{\max (P(\tilde{A}), P(\widetilde{B}))+\max \left(w_{a}, w_{b}\right)},
\end{aligned}
$$


where $S(\widetilde{A}, \widetilde{B}) \in[0,1] ; P(\widetilde{A})$ and $P(\widetilde{B})$ are defined as follows:

$$
\begin{aligned}
P(\widetilde{A})= & \sqrt{\left(a_{1}-a_{2}\right)^{2}+w_{a}^{2}}+\sqrt{\left(a_{3}-a_{4}\right)^{2}+w_{a}^{2}}+\left(a_{3}-a_{2}\right) \\
& +\left(a_{4}-a_{1}\right), \\
P(\widetilde{B})= & \sqrt{\left(b_{1}-b_{2}\right)^{2}+w_{b}^{2}}+\sqrt{\left(b_{3}-b_{4}\right)^{2}+w_{b}^{2}}+\left(b_{3}-b_{2}\right) \\
& +\left(b_{4}-b_{1}\right),
\end{aligned}
$$

where $P(\widetilde{A})$ and $P(\widetilde{B})$ denote the perimeters of the generalized trapezoidal fuzzy numbers $\widetilde{A}$ and $\widetilde{B}$, respectively. The larger the value of $S(\widetilde{A}, \widetilde{B})$ is the more the similarity is between the generalized fuzzy numbers $\widetilde{A}$ and $\widetilde{B}$.

Notice that in normalized form where height equals one $(w=1)$ this becomes

$$
S(\tilde{A}, \widetilde{B})=\left(1-\frac{\sum_{i=1}^{4}\left|a_{i}-b_{i}\right|}{4}\right) \times \frac{\min (P(\widetilde{A}), P(\widetilde{B}))}{\max (P(\widetilde{A}), P(\widetilde{B}))},
$$

where $S(\widetilde{A}, \widetilde{B}) \in[0,1] ; P(\widetilde{A})$ and $P(\widetilde{B})$ are defined as follows:

$$
\begin{aligned}
P(\widetilde{A})= & \sqrt{\left(a_{1}-a_{2}\right)^{2}+1}+\sqrt{\left(a_{3}-a_{4}\right)^{2}+1}+\left(a_{3}-a_{2}\right) \\
& +\left(a_{4}-a_{1}\right), \\
P(\widetilde{B})= & \sqrt{\left(b_{1}-b_{2}\right)^{2}+1}+\sqrt{\left(b_{3}-b_{4}\right)^{2}+1}+\left(b_{3}-b_{2}\right) \\
& +\left(b_{4}-b_{1}\right) .
\end{aligned}
$$

\section{Extended TOPSIS to the Fuzzy Environment and Using Similarity instead of Closeness Evaluation in Supplier Selection}

In this paper we follow basically the same process as given by Chen et al. [19] but when making the final evaluation and ranking we are using similarity instead of closeness criteria, and we do not use vertex method to calculate the distance between two fuzzy ratings but we use fuzzy similarity instead. About the vertex method for calculating the distance and closeness criteria see $[18,19]$. Later both are tested with numerical example next into the supplier-selection problem with extended TOPSIS.

Here we consider the importance weights of various criteria and the ratings of qualitative criteria as linguistic variables. Because linguistic assessments merely approximate the subjective judgement of decision-maker, we are using linear trapezoidal membership functions to capture the vagueness of these linguistic assessments [19,29-34]. These linguistic variables can be expressed in positive trapezoidal fuzzy numbers, as in Figures 2 and 3. The importance weight of each criterion can be either directly assigning or indirectly using pairwise comparison [35-37]. In this paper linguistic variables shown in Figures 2 and 3 are used to evaluate the importance of the criteria and the ratings of alternatives with respect to qualitative criteria. For example, the linguistic

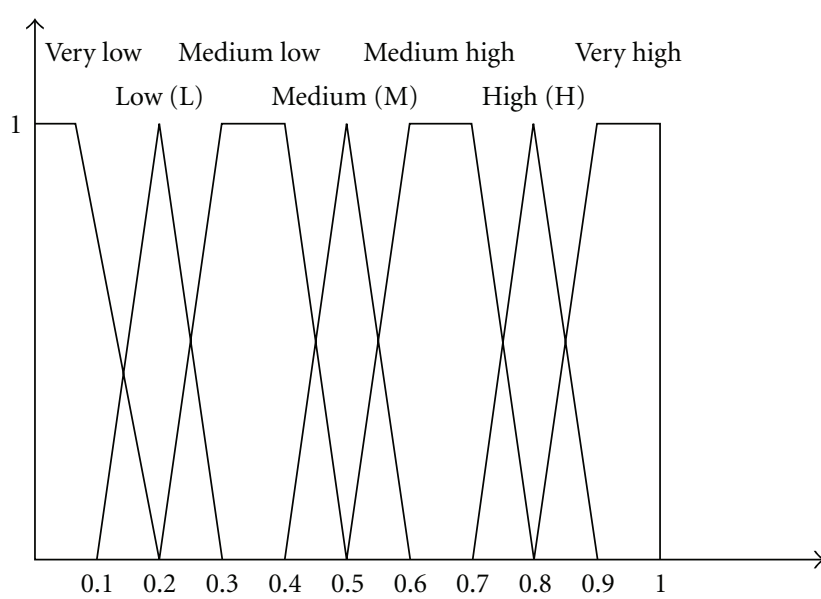

FIGURE 2: Linguistic variables for importance weight of each criterion.

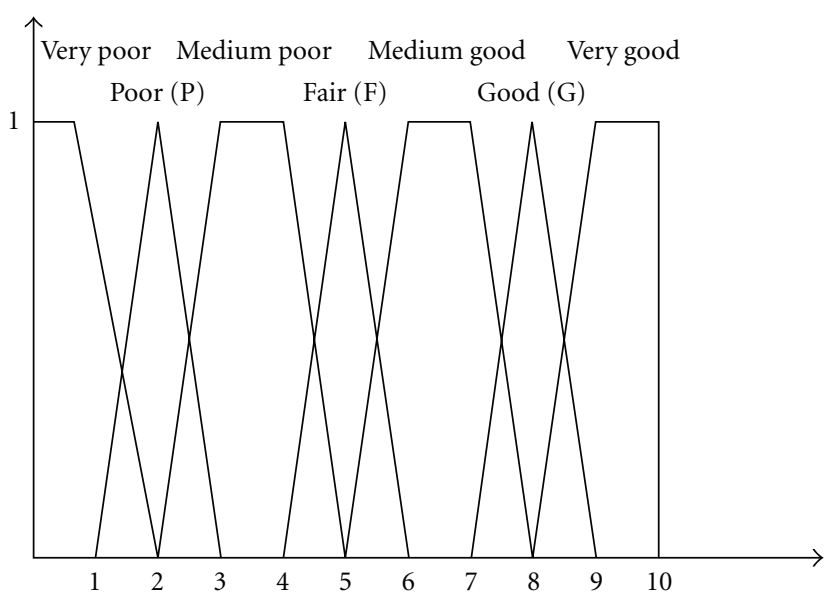

Figure 3: Linguistic variables for ratings.

variable "Medium Good" can be represented as $(5,6,7,8)$ and "Good" as $(7,8,8,9)$. Membership functions would be in these cases

$$
\begin{gathered}
\mu_{\text {Medium Good }}(x)= \begin{cases}0 & x<5, \\
\frac{x-5}{6-5} & 5 \leq x \leq 6, \\
1 & 6 \leq x \leq 7, \\
\frac{x-8}{7-8} & 7 \leq x \leq 8, \\
0 & x>8,\end{cases} \\
\mu_{\text {Good }}(x)= \begin{cases}0 & x<7, \\
\frac{x-7}{8-7} & 7 \leq x \leq 8, \\
1 & x=8, \\
\frac{x-9}{8-9} & 8 \leq x \leq 9, \\
0 & x>9 .\end{cases}
\end{gathered}
$$


Supplier selection in supply chain system is considered to be a group multiple-criteria decision-making (GMCDM) problem, which may be described by the following sets:

(i) a set of $K$ decision-makers called $E=\left\{D_{1}, D_{2}, \ldots\right.$, $\left.D_{K}\right\}$;

(ii) a set of $m$ possible suppliers called $A=\left\{A_{1}, A_{2}, \ldots\right.$, $\left.A_{m}\right\}$;

(iii) a set on $n$ criteria, $C=\left\{C_{1}, C_{2}, \ldots, C_{n}\right\}$, with which supplier performance is measured;

(iv) a set of performance ratings of $A_{i}(i=1,2, \ldots, m)$ w.r.t. criteria $C_{j}(j=1,2, \ldots, n)$ called $X=\left\{x_{i j}, i=\right.$ $1,2, \ldots, m, j=1,2, \ldots, n\}$.

Assume that a decision group has $k$ decision-makers, and the fuzzy ratings of each decision-maker $D_{k}(k=1,2, \ldots, K)$ are represented using positive trapezoidal fuzzy number $\hat{X}_{k}(k=1,2, \ldots, K)$ with membership function $\mu_{\hat{X}_{k}}(x)$. Let the fuzzy ratings of all decision-makers be trapezoidal fuzzy numbers $\hat{R}_{k}=\left(a_{k}, b_{k}, c_{k}, d_{k}\right), k=1,2, \ldots, K$. Then the aggregated fuzzy rating can be defined as

$$
\widehat{R}=(a, b, c, d)
$$

where

$$
\begin{aligned}
& a=\min _{k}\left\{a_{k}\right\} b=\frac{1}{K} \sum_{k=1}^{K} b_{k}, \\
& c=\frac{1}{K} \sum_{k=1}^{K} c_{k} d=\max _{k}\left\{d_{k}\right\} .
\end{aligned}
$$

Let the fuzzy rating and importance weight of the $k$ th decision-maker be $\hat{x}_{i j k}=\left(a_{i j k}, b_{i j k}, c_{i j k}, d_{i j k}\right)$ and $\hat{w}=\left(w_{j k 1}\right.$, $\left.w_{j k 2}, w_{j k 3}, w_{j k 4}\right) ; i=1,2, \ldots, m, j=1,2, \ldots, n$, respectively. Hence, the aggregated fuzzy ratings $\left(\hat{x}_{i j}\right)$ of alternatives with respect to each criterion can be calculated as

$$
\hat{x}_{i j}=\left(a_{i j}, b_{i j}, c_{i j}, d_{i j}\right)
$$

where

$$
\begin{aligned}
& a_{i j}=\min _{k}\left\{a_{i j k}\right\} b_{i j}=\frac{1}{K} \sum_{k=1}^{K} b_{i j k}, \\
& c_{i j}=\frac{1}{K} \sum_{k=1}^{K} c_{i j k} d_{i j}=\max _{k}\left\{d_{i j k}\right\} .
\end{aligned}
$$

In similar manner the aggregated fuzzy weight $\left(\widehat{w}_{j}\right)$ of each criterion can be calculated as

$$
\widehat{w}_{j}=\left(w_{j 1}, w_{j 2}, w_{j 3}, w_{j 4}\right),
$$

where

$$
\begin{aligned}
& w_{j 1}=\min _{k}\left\{w_{j k 1}\right\} w_{j 2}=\frac{1}{K} \sum_{k=1}^{K} w_{j k 2}, \\
& w_{j 3}=\frac{1}{K} \sum_{k=1}^{K} w_{j k 3} w_{j 4}=\max _{k}\left\{w_{j k 4}\right\} .
\end{aligned}
$$

As stated, a supplier-selection problem can be concisely expressed in matrix form as

$$
\begin{gathered}
\hat{X}=\left(\begin{array}{cccc}
\hat{x}_{11} & \hat{x}_{12} & \ldots & \hat{x}_{1 n} \\
\hat{x}_{21} & \hat{x}_{22} & \ldots & \hat{x}_{2 n} \\
\vdots & \vdots & & \vdots \\
\hat{x}_{m 1} & \hat{x}_{m 2} & \ldots & \hat{x}_{m n}
\end{array}\right), \\
\widehat{W}=\left(\widehat{w}_{1}, \widehat{w}_{2}, \ldots, \widehat{w}_{n}\right),
\end{gathered}
$$

where $\hat{x}_{i j}=\left(a_{i j}, b_{i j}, c_{i j}, d_{i j}\right)$ and $\hat{w}_{j}=\left(w_{j 1}, w_{j 2}, w_{j 3}, w_{j 4}\right)$ can be approximated by positive trapezoidal fuzzy numbers.

To avoid complexity of mathematical operations in a decision process, the linear scale transformation is used to transform the various criteria scales into comparable scales. The set of criteria can be divided into benefit criteria (the larger the rating, the greater the preference) and cost criteria (the smaller the rating, the greater the preference). Therefore, the normalized fuzzy decision matrix can be represented as

$$
\widehat{R}=\left[\widehat{r}_{i j}\right]_{m \times n},
$$

where $B$ and $C$ are the sets fo benefit criteria and cost criteria, respectively, and

$$
\begin{aligned}
& \hat{r}_{i j}=\left(\frac{a_{i j}}{d_{j}^{\oplus}}, \frac{b_{i j}}{d_{j}^{\oplus}}, \frac{c_{i j}}{d_{j}^{\oplus}}, \frac{d_{i j}}{d_{j}^{\oplus}}\right), \quad j \in B, \\
& \hat{r}_{i j}=\left(\frac{a_{j}^{\ominus}}{a_{i j}}, \frac{a_{j}^{\ominus}}{b_{i j}}, \frac{a_{j}^{\ominus}}{c_{i j}}, \frac{a_{j}^{\ominus}}{d_{i j}}\right), \quad j \in C,
\end{aligned}
$$

where $d_{j}^{\oplus}=\max _{i}\left(d_{i j}\right), j \in B$, and $a_{j}^{\ominus}=\min _{i}\left(a_{i j}\right), j \in$ $C$. This normalization method is designed to preserve the property in which the elements are normalized trapezoidal fuzzy numbers.

Considering the importance of each criterion, the weighted normalized fuzzy decision matrix can now be expressed as

$$
\widehat{V}=\left(\widehat{v}_{i j}\right)_{m \times n},
$$

where $\hat{v}_{i j}=\hat{r}_{i j}(\cdot) \hat{w}_{j}$. According to the weighted normalized fuzzy decision matrix, normalized positive trapezoidal fuzzy numbers can also approximate the elements $\hat{v}_{i j}$, for all $i, j$. Then, the fuzzy positive-ideal solution (FPIS, $A^{\oplus}$ ) and fuzzy negative-ideal solution (FNIS, $A^{\ominus}$ ) needs to be defined. Here we consider three alternatives which later will be compared.

Let fuzzy positive-ideal solution be

$$
A^{\oplus}=\left(\hat{v}_{1}^{\oplus}, \hat{v}_{2}^{\oplus}, \ldots, \hat{v}_{n}^{\oplus}\right)
$$

and fuzzy negative-ideal solution be

$$
A^{\ominus}=\left(\hat{v}_{1}^{\ominus}, \hat{v}_{2}^{\ominus}, \ldots, \hat{v}_{n}^{\ominus}\right)
$$

Criteria for choosing these was given by Chen et al. [19] as

$$
\hat{v}_{j}^{\oplus}=\max _{i}\left(v_{i j 4}\right), \quad \hat{v}_{j}^{\ominus}=\min _{i}\left(v_{i j 1}\right) .
$$


Here, for example, fuzzy positive ideal is chosen so that one finds the maximum values from fuzzy decision matrix with respect to suppliers and used the fourth value from fuzzy number ( $d$ from $\hat{A}=(a, b, c, d))$, and also replaces $a, b$, and $c$ with $d$ value (See e.g., numerical example in [19]). Fuzzy negative ideal is chosen in similar manner now just using minimum and replacing $b, c$, and $d$ with $a$. Notice that this seems a bit arbitrary since membership values at points $d$ or $a$ are zero and there we are actually using crisp numbers presented in fuzzy way instead of fuzzy numbers. Following this type of thinking one clear and simple intuitive proposal for the choice of fuzzy positive ideal would be simply the set of ones and for negative ideals a set of zeros. Third proposal which intuitively makes most sense would be that instead of replacing values for $a, b$, and $c$ with $d(\max )$ or $b, c$, and $d$ with $a$ ( $\mathrm{min}$ ) as done in [19] one simply finds the maximum and minimum fuzzy numbers from fuzzy decision matrix with respect to suppliers

$$
\hat{v}_{j}^{\oplus}=\max _{i}\left(v_{i j}\right), \quad \hat{v}_{j}^{\ominus}=\min _{i}\left(v_{i j}\right) .
$$

These three suggestions will be compared later.

Next in method described in [19] distance matrix would be calculated from $A^{\oplus}$ and $A^{\ominus}$ to each alternative supplier and after this a closeness coefficient is defined to determine the ranking order of all possible suppliers. Distances are calculated as

$$
\begin{aligned}
& d_{i}^{\oplus}=\sum_{j=1}^{n} d_{v}\left(\hat{v}_{i j}, \hat{v}_{j}^{\oplus}\right), \quad i=1,2, \ldots, m, \\
& d_{i}^{\ominus}=\sum_{j=1}^{n} d_{v}\left(\hat{v}_{i j}, \hat{v}_{j}^{\ominus}\right), \quad i=1,2, \ldots, m,
\end{aligned}
$$

where $d_{v}(;)$ is the distance measurement between two fuzzy numbers. The closeness coefficient is then calculated by

$$
C C_{i}=\frac{d_{i}^{\ominus}}{d_{i}^{\oplus}+d_{i}^{\ominus}}, \quad i=1,2, \ldots, m .
$$

Here we propose alternative way. Instead of calculating a distance matrix for both ideal solutions we can calculate similarity matrix using fuzzy similarity (see (5)) and after this simply compute average of the similarities and use this as a similarity measure to make the ranking

$$
S_{i}^{\oplus}=\frac{1}{n} \sum_{j=1}^{n} S_{v}\left(\hat{v}_{i j}, \hat{v}_{j}^{\oplus}\right) .
$$

Assessment status in Table 1 has also been used to evaluate the status of the suppliers. For this five linguistic variables with respect to the sub-intervals are defined to divide the assessment status of supplier into five classes. The decision rules for five classes are shown in Table 1 . This can be clearly also modified to suite also similarity assessment.

According to Table 1, it means the following.

(i) If $S_{i} \in[0,0.2)$, then supplier $A_{i}$ belongs to Class $\mathrm{I}$, and the assessment status of supplier $A_{i}$ is "not recommend".
TABLE 1: Approval status table.

\begin{tabular}{ll}
\hline$S$ or $C C$ value & Assessment status \\
\hline$S_{i}$ or $C C_{i} \in[0,0.2)$ & Do not recommend \\
$S_{i}$ or $C C_{i} \in[0.2,0.4)$ & Recommend with high risk \\
$S_{i}$ or $C C_{i} \in[0.4,0.6)$ & Recommend with low risk \\
$S_{i}$ or $C C_{i} \in[0.6,0.8)$ & Approved \\
$S_{i}$ or $C C_{i} \in[0.8,1.0]$ & Approved and preferred \\
\hline
\end{tabular}

(ii) If $S_{i} \in[0.2,0.4)$, then supplier $A_{i}$ belongs to Class II, and the assessment status of supplier $A_{i}$ is "recommend with high risk".

(iii) If $S_{i} \in[0.4,0.6)$, then supplier $A_{i}$ belongs to Class III, and the assessment status of supplier $A_{i}$ is "recommend with low risk".

(iv) If $S_{i} \in[0.6,0.8)$, then supplier $A_{i}$ belongs to Class IV, and the assessment status of supplier $A_{i}$ is "approved".

(v) If $S_{i} \in[0.8,1.0]$, then supplier $A_{i}$ belongs to Class V, and the assessment status of supplier $A_{i}$ is "approved and preferred to recommend".

So basically assessment status from Table 1 can be used, and in addition if any two suppliers belong to the same class of assessment status, the supplier with the highest similarity value is used to determine the rank. Notice that to these values and to approvement status given in [19] the choice of FPIS and FNIS is effecting the status considerably as later will be shown by the given example. But before going to this study proposed algorithm is summarized as follows.

Step 1. Form a committee of decision-makers, and then identify the evaluation criteria.

Step 2. Choose the appropriate linguistic variables for the importance weight of the criteria and the linguistic ratings for suppliers.

Step 3. Aggregate the weight of criteria to get the aggregated fuzzy weight $\hat{w}_{j}$ of criterion $C_{j}$, and pool the decisionmakers' ratings to get the aggregated fuzzy rating $\hat{x}_{i j}$ of supplier $A_{i}$ under criterion $C_{j}$.

Step 4. Construct the fuzzy decision matrix and the normalized fuzzy decision matrix.

Step 5. Construct weighted normalized fuzzy decision matrix.

Step 6. Determine FPIS.

Step 7. Calculate the similarity of each supplier from FPIS by calculating the similarity matrix, and then average similarity value for each supplier.

Step 8. according to the average similarity value we can get the assessment status of each supplier and determine the ranking order of all suppliers. 
TABLE 2: Importance weight of criteria from three decision-makers.

\begin{tabular}{lccc}
\hline Criteria & \multicolumn{3}{c}{ Decision-maker } \\
& $D_{1}$ & $D_{2}$ & $D_{3}$ \\
\hline$C_{1}$ & $\mathrm{H}$ & $\mathrm{H}$ & $\mathrm{H}$ \\
$C_{2}$ & $\mathrm{VH}$ & $\mathrm{VH}$ & $\mathrm{VH}$ \\
$C_{3}$ & $\mathrm{VH}$ & $\mathrm{VH}$ & $\mathrm{H}$ \\
$C_{4}$ & $\mathrm{H}$ & $\mathrm{H}$ & $\mathrm{H}$ \\
$C_{5}$ & $\mathrm{H}$ & $\mathrm{H}$ & $\mathrm{H}$ \\
\hline
\end{tabular}

\section{Numerical Example and Comparison}

Next we use numerical example to compare fuzzy TOPSIS and proposed method using fuzzy similarity and also to test how three different FPIS and FNIS criteria are effecting the results. We use the same numerical example which was given in [19] to make this comparison clear. A high-technology manufacturing company desires to select a suitable material supplier to purchase the key components of new products. After preliminary screening, five candidates $\left(A_{1}, A_{2}, A_{3}, A_{4}\right.$ and $A_{5}$ ) remain for further evaluation. A committee of three decision-makers, $D_{1}, D_{2}$, and $D_{3}$, has been formed to select the most suitable supplier. Five benefit criteria are considered:

(1) profitability of supplier $\left(C_{1}\right)$,

(2) relationship closeness $\left(C_{2}\right)$,

(3) technological capability $\left(C_{3}\right)$,

(4) conformance quality $\left(C_{4}\right)$,

(5) conflict resolution $\left(C_{5}\right)$.

The proposed method is applied to solve the problem, the computational procedure is summarized as follows:

Step 1. Three decision-makers use the linguistic weighting variables shown in Figure 2 to assess the importance of the criteria. The importance weights of the criteria can be seen in Table 2.

Step 2. Three decision-makers use the linguistic rating variables shown in Figure 3 to evaluate the ratings of suppliers with respect to each criterion. The resulting ratings are shown in Table 3.

Step 3. Convert the linguistic evaluations in Tables 2 and 3 into trapezoidal fuzzy numbers and determine the fuzzy weight of each criterion.

Step 4. Normalize the fuzzy-decision matrix.

Step 5. Build the weighted normalized fuzzy-decision matrix as in Table 4.

Step 6. Determine FPIS and FNIS. Now according to three different criteria we get three different FPIS and FNIS which are given in Table 5 .

Step 7. Calculate the similarity of each supplier by using weighted normalized fuzzy decision matrix and FPIS by
TABLE 3: Ratings of the five suppliers by three decision-makers under five criteria.

\begin{tabular}{lcccc}
\hline Criteria & Supplier & \multicolumn{3}{c}{ Decision-makers } \\
& $D_{1}$ & $D_{2}$ & $D_{3}$ \\
\hline$C_{1}$ & $A_{1}$ & $\mathrm{MG}$ & $\mathrm{MG}$ & $\mathrm{MG}$ \\
$C_{1}$ & $A_{2}$ & $\mathrm{G}$ & $\mathrm{G}$ & $\mathrm{G}$ \\
$C_{1}$ & $A_{3}$ & $\mathrm{VG}$ & $\mathrm{VG}$ & $\mathrm{G}$ \\
$C_{1}$ & $A_{4}$ & $\mathrm{G}$ & $\mathrm{G}$ & $\mathrm{G}$ \\
$C_{1}$ & $A_{5}$ & $\mathrm{MG}$ & $\mathrm{MG}$ & $\mathrm{MG}$ \\
$C_{2}$ & $A_{1}$ & $\mathrm{MG}$ & $\mathrm{MG}$ & $\mathrm{VG}$ \\
$C_{2}$ & $A_{2}$ & $\mathrm{VG}$ & $\mathrm{VG}$ & $\mathrm{VG}$ \\
$C_{2}$ & $A_{3}$ & $\mathrm{VG}$ & $\mathrm{G}$ & $\mathrm{G}$ \\
$C_{2}$ & $A_{4}$ & $\mathrm{G}$ & $\mathrm{G}$ & $\mathrm{MG}$ \\
$C_{2}$ & $A_{5}$ & $\mathrm{MG}$ & $\mathrm{G}$ & $\mathrm{G}$ \\
$C_{3}$ & $A_{1}$ & $\mathrm{G}$ & $\mathrm{G}$ & $\mathrm{G}$ \\
$C_{3}$ & $A_{2}$ & $\mathrm{VG}$ & $\mathrm{VG}$ & $\mathrm{VG}$ \\
$C_{3}$ & $A_{3}$ & $\mathrm{VG}$ & $\mathrm{VG}$ & $\mathrm{G}$ \\
$C_{3}$ & $A_{4}$ & $\mathrm{MG}$ & $\mathrm{MG}$ & $\mathrm{G}$ \\
$C_{3}$ & $A_{5}$ & $\mathrm{MG}$ & $\mathrm{MG}$ & $\mathrm{MG}$ \\
$C_{4}$ & $A_{1}$ & $\mathrm{G}$ & $\mathrm{G}$ & $\mathrm{G}$ \\
$C_{4}$ & $A_{2}$ & $\mathrm{G}$ & $\mathrm{VG}$ & $\mathrm{VG}$ \\
$C_{4}$ & $A_{3}$ & $\mathrm{VG}$ & $\mathrm{VG}$ & $\mathrm{VG}$ \\
$C_{4}$ & $A_{4}$ & $\mathrm{G}$ & $\mathrm{G}$ & $\mathrm{G}$ \\
$C_{4}$ & $A_{5}$ & $\mathrm{MG}$ & $\mathrm{MG}$ & $\mathrm{G}$ \\
$C_{5}$ & $A_{1}$ & $\mathrm{G}$ & $\mathrm{G}$ & $\mathrm{G}$ \\
$C_{5}$ & $A_{2}$ & $\mathrm{VG}$ & $\mathrm{VG}$ & $\mathrm{VG}$ \\
$C_{5}$ & $A_{3}$ & $\mathrm{G}$ & $\mathrm{VG}$ & $\mathrm{G}$ \\
$C_{5}$ & $A_{4}$ & $\mathrm{G}$ & $\mathrm{G}$ & $\mathrm{VG}$ \\
$C_{5}$ & $A_{5}$ & $\mathrm{MG}$ & $\mathrm{MG}$ & $\mathrm{MG}$ \\
\hline & & & & \\
\hline
\end{tabular}

calculating the similarity matrix, and then average similarity value for each supplier, as in Tables 6 and 7.

Step 8. According to the average similarity we get the ranking $A_{2}>A_{3}>A_{4}>A_{1}>A_{5}$. By using the approval status Table 1 we also get status approved and prefered to supplier $A_{2}$ in case we use FPIS $\mathrm{id}_{3}$. From other cases we get approval status approved. Further study about the status table can be seen in Table 7.

4.1. Comparison of Different Ideal Solutions, Usage of Fuzzy Similarity, and Closeness Coefficient. In Table 7 we have compared the approval status classes and closeness and similarity values with respect to different ideal solution criteria. As can be seen from Table 7 the ranking order is the same in each case but the assessment status of the supplier is changing depending on which ideal solution criteria we choose. If we use ideal solution criteria 1 we get as results that with closeness criteria we get that suppliers $A_{2}$ and $A_{3}$ belong to class IV and others to class III. If we use similarity we get that suppliers from $A_{1}$ to $A_{4}$ belong to class IV and $A_{5}$ to class III. If we use the second ideal solution criteria we get the results other way around. With closeness coefficient suppliers from $A_{1}$ to $A_{4}$ belong to class IV and with similarity $A_{2}$ and $A_{3}$ belong to class IV and others to class III. Then if we choose 
TABLE 4: Weighted normalized fuzzy decision matrix.

\begin{tabular}{cccccc}
\hline & $C_{1}$ & $C_{2}$ & $C_{3}$ & $C_{4}$ & $C_{5}$ \\
\hline$A_{1}$ & $(0.35,0.48,0.56,0.72)$ & $(0.4,0.63,0.8,1)$ & $(0.49,0.7,0.74,0.9)$ & $(0.49,0.64,0.64,0.81)$ & $(0.49,0.64,0.64,0.81)$ \\
$A_{2}$ & $(0.49,0.64,0.64,0.81)$ & $(0.64,0.81,1,1)$ & $(0.56,0.78,0.93,1)$ & $(0.49,0.7,0.74,0.9)$ & $(0.56,0.72,0.8,0.9)$ \\
$A_{3}$ & $(0.49,0.7,0.75,0.9)$ & $(0.56,0.75,0.87,1)$ & $(0.49,0.76,0.86,1)$ & $(0.56,0.72,0.8,0.9)$ & $(0.49,0.66,0.7,0.9)$ \\
$A_{4}$ & $(0.49,0.64,0.64,0.81)$ & $(0.4,0.66,0.77,0.9)$ & $(0.35,0.58,0.68,0.9)$ & $(0.49,0.64,0.64,0.81)$ & $(0.49,0.66,0.7,0.9)$ \\
$A_{5}$ & $(0.35,0.48,0.56,0.72)$ & $(0.4,0.66,0.77,0.9)$ & $(0.35,0.52,0.65,0.8)$ & $(0.35,0.53,0.59,0.81)$ & $(0.35,0.48,0.56,0.72)$ \\
\hline
\end{tabular}

TABle 5: Fuzzy positive-ideal solutions and fuzzy negative-ideal solutions according to different criteria.

\begin{tabular}{cccccc}
\hline$A_{\mathrm{id} 1}^{\oplus}$ & $(0.9,0.9,0.9,0.9)$ & $(1,1,1,1)$ & $(1,1,1,1)$ & $(0.9,0.9,0.9,0.9)$ & $(0.9,0.9,0.9,0.9)$ \\
$A_{\mathrm{id} 1}^{\ominus}$ & $(0.35,0.35,0.35,0.35)$ & $(0.4,0.4,0.4,0.4)$ & $(0.35,0.35,0.35,0.35)$ & $(0.35,0.35,0.35,0.35)$ & $(0.35,0.35,0.35,0.35)$ \\
\hline$A_{\mathrm{id} 2}^{\oplus}$ & $(1,1,1,1)$ & $(1,1,1,1)$ & $(1,1,1,1)$ & $(1,1,1,1)$ & $(1,1,1,1)$ \\
$A_{\mathrm{id} 2}^{\ominus}$ & $(0,0,0,0)$ & $(0,0,0,0)$ & $(0,0,0,0)$ & $(0,0,0,0)$ & $(0,0,0,0)$ \\
\hline$A_{\mathrm{id} 3}^{\oplus}$ & $(0.49,0.69,0.75,0.9)$ & $(0.64,0.81,1,1)$ & $(0.56,0.78,0.93,1)$ & $(0.56,0.72,0.8,0.9)$ & $(0.56,0.72,0.8,0.9)$ \\
$A_{\mathrm{id} 3}^{\ominus}$ & $(0.35,0.48,0.56,0.72)$ & $(0.4,0.63,0.77,0.9)$ & $(0.35,0.52,0.65,0.8)$ & $(0.35,0.53,0.59,0.81)$ & $(0.35,0.48,0.56,0.72)$ \\
\hline
\end{tabular}

TABLE 6: Similarities between FPIS and weighted normalized fuzzy decision matrix.

\begin{tabular}{|c|c|c|c|c|c|c|}
\hline & $C_{1}$ & $C_{2}$ & $C_{3}$ & $C_{4}$ & $C_{5}$ & Average \\
\hline$S\left(A_{1}, A_{\mathrm{id} 1}^{\oplus}\right)$ & 0.54 & 0.56 & 0.61 & 0.67 & 0.67 & 0.61 \\
\hline$S\left(A_{2}, A_{\mathrm{id} 1}^{\oplus}\right)$ & 0.69 & 0.73 & 0.68 & 0.69 & 0.74 & 0.70 \\
\hline$S\left(A_{3}, A_{\mathrm{id} 1}^{\oplus}\right)$ & 0.69 & 0.66 & 0.64 & 0.74 & 0.68 & 0.68 \\
\hline$S\left(A_{4}, A_{\mathrm{id} 1}^{\oplus}\right)$ & 0.67 & 0.56 & 0.51 & 0.67 & 0.68 & 0.62 \\
\hline$S\left(A_{5}, A_{\mathrm{id} 1}^{\oplus}\right)$ & 0.54 & 0.56 & 0.48 & 0.57 & 0.54 & 0.54 \\
\hline$S\left(A_{1}, A_{\mathrm{id} 2}^{\oplus}\right)$ & 0.46 & 0.56 & 0.61 & 0.58 & 0.58 & 0.56 \\
\hline$S\left(A_{2}, A_{\mathrm{id} 2}^{\oplus}\right)$ & 0.58 & 0.73 & 0.68 & 0.61 & 0.65 & 0.65 \\
\hline$S\left(A_{3}, A_{\mathrm{id} 2}^{\oplus}\right)$ & 0.61 & 0.66 & 0.64 & 0.65 & 0.59 & 0.63 \\
\hline$S\left(A_{4}, A_{\mathrm{id} 2}^{\oplus}\right)$ & 0.58 & 0.56 & 0.51 & 0.58 & 0.59 & 0.56 \\
\hline$S\left(A_{5}, A_{\mathrm{id} 2}^{\oplus}\right)$ & 0.46 & 0.56 & 0.48 & 0.48 & 0.46 & 0.49 \\
\hline$S\left(A_{1}, A_{\mathrm{id} 3}^{\oplus}\right)$ & 0.81 & 0.79 & 0.86 & 0.88 & 0.88 & 0.84 \\
\hline$S\left(A_{2}, A_{\mathrm{id} 3}^{\oplus}\right)$ & 0.9 & 1 & 1 & 0.95 & 1 & 0.97 \\
\hline$S\left(A_{3}, A_{\mathrm{id} 3}^{\oplus}\right)$ & 1 & 0.93 & 0.95 & 1 & 0.93 & 0.96 \\
\hline$S\left(A_{4}, A_{\mathrm{id} 3}^{\oplus}\right)$ & 0.90 & 0.8 & 0.79 & 0.88 & 0.93 & 0.86 \\
\hline$S\left(A_{5}, A_{\mathrm{id} 3}^{\oplus}\right)$ & 0.81 & 0.8 & 0.76 & 0.80 & 0.78 & 0.79 \\
\hline
\end{tabular}

the third criteria which seems most realistic and is truly using fuzzy trapezoidal numbers instead of presenting crisp numbers in a fuzzy way we get quite high assessments with similarity, now suppliers $A_{1}$ to $A_{4}$ belonging to class $\mathrm{V}$ and $A_{5}$ belonging to class IV. If we look at the closeness coefficient we get surprisingly different results as suppliers $A_{2}$ and $A_{3}$ get assessment status belonging to class $\mathrm{V}$ and $A_{4}$ belonging to class III, $A_{1}$ to class II, and $A_{5}$ to class I. When we consider how realistic these assessment status ratings are we first need to look to the decision-makers assessments. As can be seen from Table 3 decision-makers gave quite good ratings for the suppliers. There were several ratings as "very good" and even worse ratings were only "medium good". Notice that even "Fair" which was in the middle was never used not to mention "Poor". So decision-makers evaluated these suppliers with quite high ratings. This speaks to advantage of criteria three from fuzzy positive-ideal solution and the usage of similarity instead of closeness coefficient since with closeness coefficient rating, for example, for supplier $A_{5}$ would have been "do not recommend" even though all the decision-makers gave at least "medium good" rating for this supplier.

Having pointed this one also should notice that this approval status Table 1 was build more for closeness coefficient and using FPIS and FNIS with criteria 1; so to suggest appropriate approval status table from these experiments one could suggest to use similarity, criteria 3 for FPIS, and approval status given in Table 8 .

From this approval status Table 8 assessments status would be approved and prefered for $A_{2}$ and $A_{3}$ and approved for $A_{4}$ and $A_{1}$ and recommended with low risk for $A_{5}$. If one prefers to use FPIS criteria 2 and similarity then approval status Table 1 would be preferred. This experiment clearly shows that one should not just blindly use approval status table given in [19] (see also Table 1) but also consider the situation at hand and preferably even test different FPIS and FNIS criteria to get a better understanding about the supplier evaluation. 
TABLE 7: Comparison of closeness coefficient $\left(C C_{i}\right)$ and similarity values $\left(S_{i}\right)$ in approval status table with different ideal solution criteria.

\begin{tabular}{lccccccccccccc}
\hline Supplier & $C C_{\mathrm{id} 1}$ & Class & $S_{\mathrm{id} 1}$ & Class & $C C_{\mathrm{id} 2}$ & Class & $S_{\mathrm{id} 2}$ & Class & $C C_{\text {id3 }}$ & Class & $S_{\mathrm{id} 3}$ & Class \\
\hline$A_{1}$ & 0.496 & III & 0.608 & IV & 0.632 & IV & 0.555 & III & 0.364 & II & 0.842 & V \\
$A_{2}$ & 0.642 & IV & 0.701 & IV & 0.728 & IV & 0.648 & IV & 0.884 & V & 0.969 & V \\
$A_{3}$ & 0.622 & IV & 0.682 & IV & 0.715 & IV & 0.630 & IV & 0.808 & V & 0.961 & V \\
$A_{4}$ & 0.511 & III & 0.617 & IV & 0.643 & IV & 0.564 & III & 0.414 & III & 0.859 & V \\
$A_{5}$ & 0.404 & III & 0.539 & III & 0.570 & III & 0.487 & III & 0.015 & I & 0.790 & IV \\
\hline
\end{tabular}

TABLE 8: Approval status table.

\begin{tabular}{ll}
\hline Similarity value & Assessment status \\
\hline$S_{i} \in[0,0.5)$ & Do not recommend \\
$S_{i} \in[0.5,0.7)$ & Recommend with high risk \\
$S_{i} \in[0.7,0.8)$ & Recommend with low risk \\
$S_{i} \in[0.8,0.9)$ & Approved \\
$S_{i} \in[0.9,1.0]$ & Approved and preferred \\
\hline
\end{tabular}

\section{Conclusions and Discussion}

In this paper a new approach is proposed to deal with the supplier selection problem in an uncertain environment. This problem to be solved is MCDM problem. In conventional MCDM methods, the ratings and the weights of attributes must be known precisely [17, 38]. This requirement however is not met in many situations since decision-makers judgements are often uncertain and cannot be estimated by an exact numerical value. This leads to the fact that the problem of selecting suppliers has many uncertainties and becomes more difficult. Fuzzy methods provides a solution for supplier selection problem [19]. However in evaluation of the supplier the evaluation depends heavily on the selection of appropriate fuzzy positive-ideal solution (FPIS) and fuzzy negative-ideal solution (FNIS). In this paper we have proposed three different criteria for selecting FPIS and FNIS and studied how they affect supplier evaluation. Also a new proposal to fuzzy supplier evaluation and selection is given. This method uses fuzzy similarity to make supplier selection and evaluation. By using this method less computations are needed to supplier selection problem, and we do not need to calculate FNIS anymore when using similarity, but we can simply use given fuzzy similarity measure to calculate similarities between FPIS and suppliers. This simplifies the model and makes computations a bit easier. Also new assessment status Table 8 is proposed when similarity and third FPIS criteria are used. The experimental results show that the proposed approach is reliable and reasonable.

\section{References}

[1] R. Monczka, R. Trent, and R. Handfield, Purchasing and Supply Chain Management, South-Western College Publishing, New York, NY, USA, 1998.

[2] J. Choi, S. X. Bai, J. Geunes, and H. E. Romeijn, "Manufacturing delivery performance for supply chain management,"
Mathematical and Computer Modelling, vol. 45, no. 1-2, pp. 11-20, 2007.

[3] K. N. Thompson, "Vendor profile analysis," Journal of Purchasing and Materials Management, vol. 26, no. 1, pp. 11-18, 1990.

[4] E. Timmerman, "An approach to vendor performance evaluation," Journal of Purchasing and Supply Management, vol. 1, pp. 27-32, 1986.

[5] G. Barbarosoğlu and T. Yazgaç, "An application of the analytic hierarchy process to the supplier selection problem," Production and Inventory Management Journal, vol. 38, no. 1, pp. 1421, 1997.

[6] R. Narasimhan, "An analytic approach to supplier selection," Journal of Purchasing and Supply Management, vol. 1, pp. 2732, 1983.

[7] J. Sarkis and S. Talluri, "A model for strategic supplier selection," in Proceedings of the 9th International IPSERA Conference, pp. 652-661, London, Canada, May 2000.

[8] R. M. Monezka and S. J. Trecha, "Cost-based supplier performance evaluation," Journal of Purchasing and Materials Management, vol. 24, no. 2, pp. 2-7, 1998.

[9] D. L. Smytka and M. W. Clemens, "Total cost supplier selection model: a case study," International Journal of Purhasing and Materials Management, vol. 29, no. 1, pp. 42-49, 1993.

[10] F. P. Buffa and W. M. Jackson, "A goal programming model for purchase planning," Journal of Purchasing and Material Management, vol. 19, no. 3, pp. 27-34, 1983.

[11] S. S. Chaudhry, F. G. Forst, and J. L. Zydiak, "Vendor selection with price breaks," European Journal of Operational Research, vol. 70, no. 1, pp. 27-34, 1993.

[12] S. Alonso, E. Herrera-Viedma, F. Chiclana, and F. Herrera, "A web based consensus support system for group decision making problems and incomplete preferences," Information Sciences, vol. 180, no. 23, pp. 4477-4495, 2010.

[13] F. Chiclana, E. Herrera-Viedma, and S. Alonso, "A note on two methods for estimating missing pairwise preference values," IEEE Transactions on Systems, Man, and Cybernetics B, vol. 39, no. 6, pp. 1628-1633, 2009.

[14] B. Donaldson, "Supplier selection criteria on the service dimension. Some empirical evidence," European Journal of Purchasing and Supply Management, vol. 1, no. 4, pp. 209-217, 1994.

[15] L. De Boer, L. Van Der Wegen, and J. Telgen, "Outranking methods in support of supplier selection," European Journal of Purchasing and Supply Management, vol. 4, no. 2-3, pp. 109118, 1998.

[16] S. H. Ghodsypour and C. O’Brien, “A decision support system for supplier selection using an integrated analytic hierarchy process and linear programming," International Journal of Production Economics, vol. 56-57, pp. 199-212, 1998.

[17] C. L. Hwang and K. P. Yoon, Multiple Attirubtes Decision Making: Methods and Applications, Springer, Berlin, Germany, 1981. 
[18] C. T. Chen, "Extensions of the TOPSIS for group decisionmaking under fuzzy environment," Fuzzy Sets and Systems, vol. 114, no. 1, pp. 1-9, 2000.

[19] C. T. Chen, C. T. Lin, and S. F. Huang, "A fuzzy approach for supplier evaluation and selection in supply chain management," International Journal of Production Economics, vol. 102, no. 2, pp. 289-301, 2006.

[20] S. H. Wei and S. M. Chen, "A new approach for fuzzy risk analysis based on similarity measures of generalized fuzzy numbers," Expert Systems with Applications, vol. 36, pp. 589598, 2009.

[21] L. Zadeh, "Fuzzy sets," Information and Control, vol. 8, pp. 338-353, 1965.

[22] L. Zadeh, "Similarity relations and fuzzy orderings," Information Sciences, vol. 3, pp. 177-200, 1971.

[23] S. J. Chen and S. M. Chen, "A new method to measure the similarity between fuzzy numbers," in Proceedings of the 10th International Conference on Fuzzy Systems, Melbourne, Australia, 2001.

[24] S. M. Chen, "New methods for subjective mental workload assessment and fuzzy risk analysis," Cybernetics and Systems, vol. 27, no. 5, pp. 449-472, 1996.

[25] C. H. Hsieh and S. H. Chen, "Similarity of generalized fuzzy numbers with graded mean integration representation," in Proceedings of the 8th International Fuzzy Systems Association World Congress, vol. 2, pp. 551-555, Taipei, Taiwan, 1999.

[26] H. S. Lee, "Optimal aggregation method for fuzzy opinions of group decision," in Proceedings of the IEEE International Conference on Systems, Man, and Cybernetics, vol. 3, pp. 314319, October 1999.

[27] S. H. Chen, "Ranking generalized fuzzy number with graded mean integration," in Proceedings of the 8th International Fuzzy Systems Association World Congress, vol. 2, pp. 899-902, Taipei, Taiwan, 1999.

[28] D. Dubois and H. Prade, Fuzzy Sets and Systems: Theory and Applications, Academic Press, New York, NY, USA, 1980.

[29] M. Delgado, F. Herrera, E. Herrera-Viedma, and L. Martínez, "Combining numerical and linguistic information in group decision making," Information Sciences, vol. 107, no. 1-4, pp. 177-194, 1998.

[30] F. Herrera and E. Herrera-Viedma, "Linguistic decision analysis: steps for solving decision problems under linguistic information," Fuzzy Sets and Systems, vol. 115, no. 1, pp. 67$82,2000$.

[31] F. Herrera, E. Herrera-Viedma, and J. L. Verdegay, "A model of consensus in group decision making under linguistic assessments," Fuzzy Sets and Systems, vol. 78, no. 1, pp. 73-87, 1996.

[32] I. J. Pérez, F. J. Cabrerizo, and E. Herrera-Viedma, "Group decision making problems in a linguistic and dynamic context," Expert Systems with Applications, vol. 38, no. 3, pp. 1675$1688,2011$.

[33] F. J. Cabrerizo, I. J. Pérez, and E. Herrera-Viedma, "Managing the consensus in group decision making in an unbalanced fuzzy linguistic context with incomplete information," Knowledge-Based Systems, vol. 23, no. 2, pp. 169-181, 2010.

[34] F. J. Cabrerizo, S. Alonso, and E. Herrera-Viedma, "A consensus model for group decision making problems with unbalanced fuzzy linguistic information," International Journal of Information Technology and Decision Making, vol. 8, no. 1, pp. 109-131, 2009.

[35] R. L. Cook, "Expert systems in purchasing applications and development," International Journal of Purchasing and Management, vol. 18, pp. 20-27, 1992.
[36] I. J. Pérez, F. J. Cabrerizo, and E. Herrera-Viedma, "A mobile decision support system for dynamic group decisionmaking problems," IEEE Transactions on Systems, Man, and Cybernetics A, vol. 40, no. 6, Article ID 5466045, pp. 12441256, 2010.

[37] I. J. Perez, F. J. Cabrerizo, and E. Herrera-Viedma, “A Mobile Group Decision Making Model for Heterogeneous Information and Changeable Decision Contexts," International Journal of Uncertainty, Fuzziness and Knowledge-Based Systems, vol. 19, no. 1, pp. 33-52, 2011.

[38] M. Delgado, J. L. Verdegay, and M. A. Vila, "Linguistic decision-making models," International Journal of Intelligent Systems, vol. 7, no. 5, pp. 479-492, 1992. 

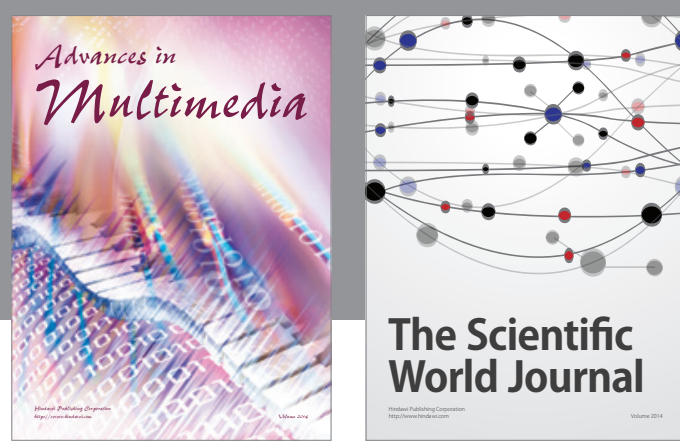

The Scientific World Journal
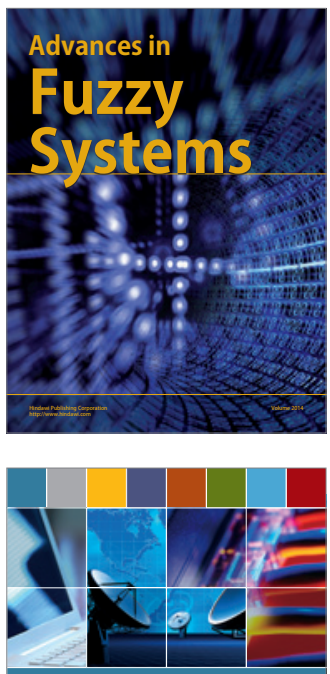

Computer Networks and Communications
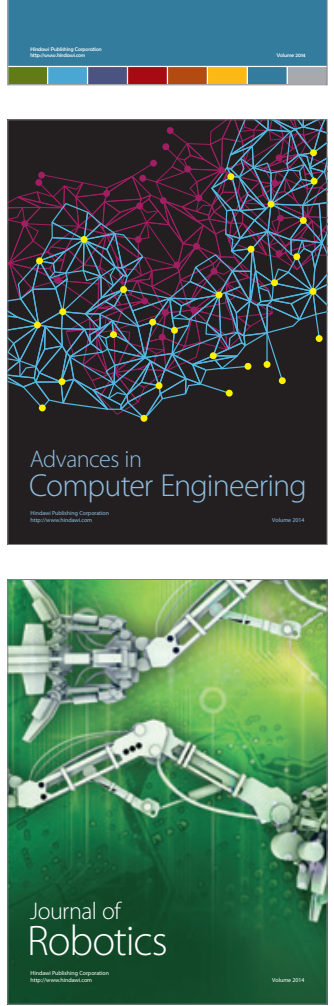
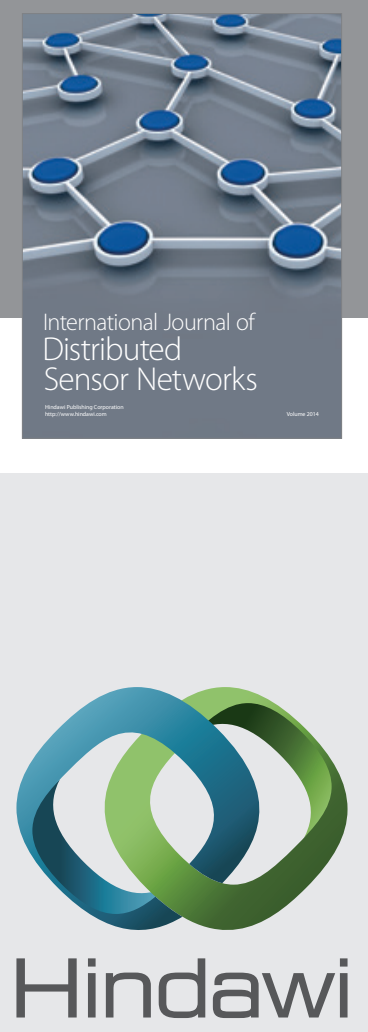

Submit your manuscripts at

http://www.hindawi.com
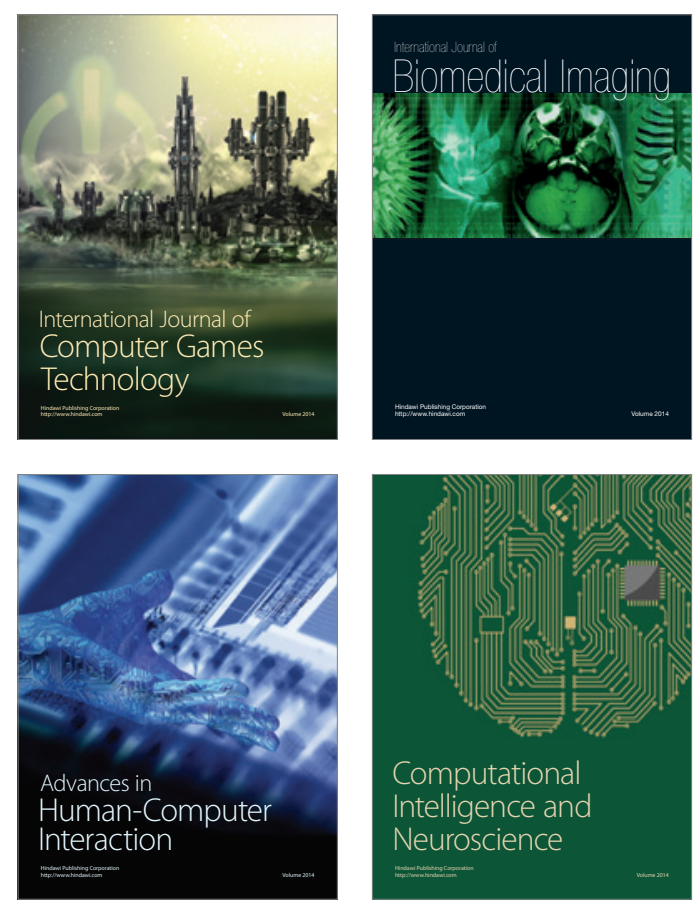
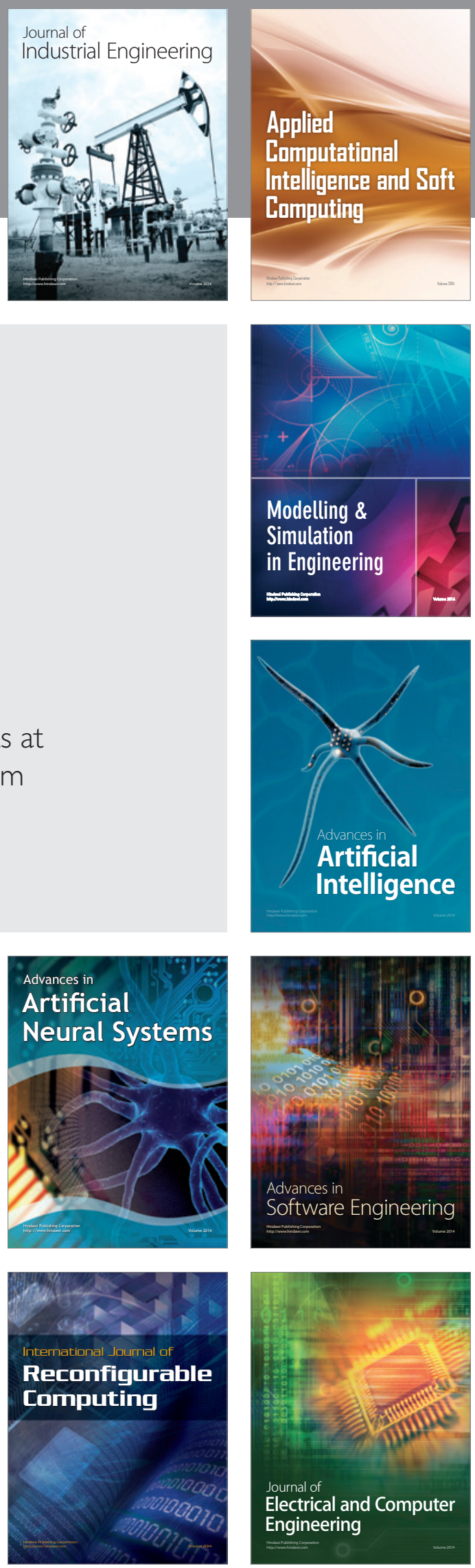